INPLASY

PROTOCOL

To cite: Mao et al. Reinfection rates among patients who previously infected for COVID-19: a systematic review and meta-analysis. Inplasy protocol 202160104. doi: 10.37766/inplasy2021.6.0104

Received: 28 June 2021

Published: 28 June 2021

Corresponding author:

Feng Sun

sunfeng@bjmu.edu.cn

Author Affiliation:

Peking University School of

Public Health.

Support: None.

Review Stage at time of this submission: Ongoing.

Conflicts of interest: None declared.

\section{Reinfection rates among patients who previously infected for COVID-19: a systematic review and meta-analysis}

\author{
Mao, Y1; Wang, W2; Ma, J3; Sun, F4.
}

Review question / Objective: Asymptomatic or symptomatic coronavirus disease 2019 infection is followed by gradual detection of reinfection. The protection offered by prior infection among patients with severe acute respiratory syndrome coronavirus 2 (SARS-CoV-2) is unclear. We aimed to assess the risk of SARS-CoV-2 reinfections and the protection afforded from the initial infection.

Condition being studied: COVID-19.

Information sources: A comprehensive search was conducted using PubMed, EMBASE, Cochrane, Scopus, Web of Science, and ClinicalTrials.gov. Manually search the WHO report and the references in the retrieved articles to ensure the identification of studies that were not found in the initial literature search.

INPLASY registration number: This protocol was registered with the International Platform of Registered Systematic Review and Meta-Analysis Protocols (INPLASY) on 28 June 2021 and was last updated on 28 June 2021 (registration number INPLASY202160104).

\section{INTRODUCTION}

Review question / Objective: Asymptomatic or symptomatic coronavirus disease 2019 infection is followed by gradual detection of reinfection. The protection offered by prior infection among patients with severe acute respiratory syndrome coronavirus 2
(SARS-CoV-2) is unclear. We aimed to assess the risk of SARS-CoV-2 reinfections and the protection afforded from the initial infection.

Condition being studied: Reinfection patients with COVID-19. 


\section{METHODS}

Participant or population: Reinfection patients with COVID-19.

Intervention: COVID-19.

Comparator: Not applicable.

Study designs to be included: Original research with any type of study designs, containing the total number of initial positive patients.

Eligibility criteria: Studies were selected based on the following inclusion criteria: (1) Studies about the definition of reinfection met CDC standards; (2) Original research with any type of study designs, containing the total number of initial positive patients. The exclusion criteria were: (1) There were no full texts of the studies; (2) Patients with Middle East respiratory syndrome coronavirus or other serotypes of SARSCoV infection.

Information sources: A comprehensive search was conducted using PubMed, EMBASE, Cochrane, Scopus, Web of Science, and ClinicalTrials.gov. Manually search the WHO report and the references in the retrieved articles to ensure the identification of studies that were not found in the initial literature search.

Main outcome(s): Reinfection rate, symptomatic reinfection rate, protection offered by prior infection against reinfection, protection offered by prior infection against symptomatic reinfection, reinfection rate of high-risk population.

Quality assessment / Risk of bias analysis: The Newcastle-Ottawa Scale was used to evaluate the bias of the cohort studies, and the JBI critical appraisal tool was used to evaluate the bias of the cross-sectional and ecological studies.

Strategy of data synthesis: We used a random-effects model to obtain a pooled estimate and $95 \%$ confidence interval (Cl) for the reinfection rate after recovery from COVID-19, and constructed an arcsine transformation to stabilize the variance. The Cochrane 12 statistic was used to estimate the statistical heterogeneity among the included studies. A cumulative meta-analysis was carried out to analyze whether the results tended to stabilize as the sample size increases.

Subgroup analysis: We hypothesized that different gender, study type, continent, disease severity, age, and comorbidities may have an impact on reinfection rate.

Sensitivity analysis: We performed a sensitivity analysis to test the reliability of the results.

Country(ies) involved: China.

Keywords: COVID-19, reinfection, SARSCoV-2, rate.

Contributions of each author:

Author 1 - Yinjun Mao.

Author 2 - Weiwei Wang.

Author 3 - Jun Ma.

Author 4 - Feng Sun. 